\title{
Qualitätssicherung in der interventionellen Neuroradiologie
}

\author{
W. Weber $\cdot$ A. Berlis
}

\section{Quality assurance in interventional neuroradiology}

Blickt man auf den Fortschritt in der interventionellen Neuroradiologie während der letzten 50 Jahre zurück, ist sicher der wichtigste Meilenstein die Entwicklung der ablösbaren Platinmikrospiralen (,,coils") zur Aneurysmabehandlung Anfang der 1990er Jahre. Zahlenmäßig hat die endovaskuläre Behandlung von Hirnarterienaneurysmen seitdem enorm zugenommen. Wie in den entsprechenden Abhandlungen dieser Festschrift nachzulesen, haben Weiterentwicklungen im Bereich der endovaskulären Behandlung von Hirnarterienaneurysmen wie Ballons, Stents und Verbesserungen der Coils selbst, diesen Effekt, insbesondere in den letzten 10 Jahren, verstärkt. Aber auch auf den anderen neurointerventionellen Gebieten gab und gibt es enorme Fortschritte. Beispielsweise bei der Behandlung von arteriovenösen Malformationen (AVM) und duralen AV-Fisteln mit der Entwicklung des flüssigen Embolisats Onyx $^{\mathrm{TM}}$. Bessere Führungs- und Mikrokatheter sowie die Verbesserung der Mikrodrähte haben die sichere Behandlung immer komplexerer Erkrankungen möglich gemacht. Der aktuell letzte Meilenstein ist die Entdeckung der mechanischen Rekanalisation von Hirngefäßen mithilfe von ,stent

\author{
W. Weber $(\bowtie)$ \\ Radiologie, Neuroradiologie und Nuklearmedizin, \\ Universitätsklinikum Knappschaftskrankenhaus Bochum GmbH, \\ In der Schornau 23-25, \\ 44892 Bochum, Deutschland \\ E-Mail: werner.weber@kk-bochum.de \\ A. Berlis \\ Klinik für Diagnostische und Interventionelle Neuroradiologie, \\ Klinikum Augsburg, \\ Stenglinstraße 2, \\ 86356 Augsburg, Deutschland
}

retrievern" allein oder in Kombination mit geeigneten Aspirationskathetern. Die Evidenz dieser Methode wurde kürzlich in mehreren Studien belegt [1-4].

Steigende Interventionszahlen, Interventionsarten mit immer mehr und komplexeren Materialien, bei steigender Anzahl der interventionell tätigen Institute, machen es immer schwieriger, einen Überblick über die Behandlungen zu behalten, die im Verantwortungsbereich der Deutschen Gesellschaft für Neuroradiologie (DGNR) durchgeführt worden sind und werden. Die Frage zu beantworten: Was tun wir eigentlich, mit welchem Erfolg, wird immer schwieriger. Jeder für sich mag das für sein Institut beantworten können, aber schon der Vergleich mit anderen stößt an Grenzen, weil hierzu Daten fehlen. Die Darstellung unseres medizinischen Handelns gegenüber unseren Patienten, unseren klinischen Partnern, den wirtschaftlich in unserem Krankenhaus Verantwortlichen und gegenüber den Kostenträgern in unserem Gesundheitssystem wird immer wichtiger, um die Zukunft der interventionell neuroradiologischen Verfahren in unseren Händen zu sichern. Wir glauben zu wissen, dass wir Gutes tun. Es wird aber immer bedeutender, dass die Anderen das sehen und belegt bekommen. Wir müssen unsere medizinischen Handlungen erfassen, darstellen und kommunizieren und zwar nicht als einzelne Institution, sondern als Gesellschaft. Qualitätssicherung kann ein wertvolles berufspolitisch mächtiges Werkzeug sein, dies z. B. auf dem Gebiet der Fort- und Weiterbildung oder als Lieferant von Informationen zu ökonomischen und wissenschaftlichen Aspekten der interventionellen Radiologie. Zum Teil ist die Teilnahme an Qualitätssicherung(QS)-Programmen bereits mit der Vergütung unserer Behandlungen verbindlich verknüpft [AQUA-Institut - „,carotid endarterectomy“ (CEA) und „,carotid artery stenting“ (CAS)].

Ein geeignetes Instrument zur QS in der interventionellen Radiologie und Neuroradiologie ist das DeGIR-QS-Re- 
gister (DeGIR: Deutsche Gesellschaft für Interventionelle Radiologie und minimal-invasive Therapie) derzeit auf der Basis der internetbasierten Software der BQS Institut für Qualität \& Patientensicherheit GmbH. Die Zahl der freiwillig teilnehmenden Institute steigt kontinuierlich an und hat durch den Beitritt der DGNR Ende 2012 enormen Schub erhalten. Hier werden die einzelnen Interventionen bezüglich ihrer Art, Durchführung und Ergebnis erfasst. Auch Komplikationen und Strahlenschutzaspekte können eingegeben und sowohl für das einzelne Institut als auch im Vergleich mit allen Instituten erfasst und verglichen werden. Verbunden mit der Teilnahme der Institute am DeGIR-QSRegister kann neben persönlichen Zertifikaten, eine Zertifizierung zur Ausbildungsstätte insgesamt oder bezogen auf einzelne Module erfolgen. Informationen hierzu finden sich auf: www.dgnr.de und www.degir.de.

Ein strukturiertes Ausbildungsprogramm in interventioneller Radiologie (IR), an dessen Ende eine DeGIR-Zertifizierung steht, wurde in Kooperation mit der Akademie für Fort- und Weiterbildung der Deutschen Röntgengesellschaft 2009 von Peter Reimer (Karlsruhe) und Peter Landwehr (Hannover) vorgelegt. Dies sah ein Stufenkonzept mit Basisqualifizierung (Stufe 1) und Spezialisierung in IR vor. Auf dem Berufsverbandstreffen in Würzburg im Mai 2010 wurde erstmals durch Ansgar Berlis und Hartmut Brückmann auf dieses Ausbildungsprogramm hingewiesen und eine ähnliche Struktur für die Neuroradiologie gefordert. Zunächst wurde in Richtung Zusatzweiterbildung in interventioneller Neuroradiologie innerhalb des Schwerpunkts diskutiert. Ein Jahr später auf dem Berufsverbandstreffen in Darmstadt (Mai 2011) wurde der Vorschlag einer Zertifizierung „Interventionelle Neuroradiologie (INR)“ als „NeuroModul E“ nach Muster der DeGIR formuliert. Es erging der Auftrag der DGNR, zusammen mit der DeGIR ein entsprechendes gemeinsames Konzept zu entwickeln. Als Vertreter der DGNR wurden Ansgar Berlis und Werner Weber bestimmt, die mit Peter Reimer und Peter Landwehr von der DeGIR das heute gültige Konzept mit 2 Neuromodulen erarbeiteten. Die neuroradiologischen Qualitätsmodule E für die gefäßeröffnenden Verfahren und $\mathrm{F}$ für die gefäßverschließenden Verfahren wurden im September 2012 den bestehenden Modulen der Radiologie (A-D) hinzugefügt.

In die DeGIR-Datenbank konnten neuroradiologische Interventionen schon seit vielen Jahren eingegeben werden. Hierfür waren anfänglich Lothar Heuser und Isabel Wanke zuständig. Die Ansprüche an das System änderten sich mit der Einführung der Neuromodule, da die Neurointerventionen nun wesentlich differenzierter abgebildet sein mussten. Hinzu kam, dass die Teilnahme an dem BQS-System Voraussetzung für das DeGIR-DGNR-Ausbildungsstätten-Zertifikat wurde. Vonseiten der DGNR liegt die Verantwortung seit 2011 bei den Autoren dieses Beitrags.
Betrachtet man die Entwicklung nur der letzten 4 Jahre, hat sich die Zahl der teilnehmenden Institute am BQS von $158 \mathrm{im}$ Jahr 2010 auf 255 im Jahr 2013 fast verdoppelt. Noch imposanter ist die Zunahme der eingegebenen Interventionen, von $51.769 \mathrm{im}$ Jahr 2010 auf insgesamt 115.190 im Jahr 2013.

Für das Jahr 2013 entfielen auf die Neuroradiologie 4873 Eingriffe zur mechanischen Rekanalisation von Hirnarterien (Rang 5) und 3194 Eingriffe zur endovaskulären Behandlung von Hirnarterienaneurysmen (Rang 9). Berücksichtigt man die Komplexität der erfassten interventionellen Verfahren, stehen die interventionellen Schlaganfallbehandlungen zahlenmäßig vor den venösen Rekanalisationen und die Aneurysmabehandlungen vor den transarteriellen Chemoembolisationen. Erste Publikationen aus diesem Datenmaterial zur arteriellen Verschlusskrankheit und zum Aortenaneurysma sind erschienen [5, 6]. Eine Publikation mit Auswertung der eingegebenen Daten zu den endovaskulären Behandlungen der Hirnarterienaneurysmen ist in Vorbereitung.

Eine Neuerung für 2015 ist die nun mögliche Erfassung von „new devices“. Neu ist die namentliche Erfassung neuer Interventionsmaterialien, um intern einen Überblick über Erfolg und möglichen Misserfolg einzelner Produkte zu erhalten. Auch hier, wie in der gesamten Datenbank, bleiben alle weiteren Informationen anonym und die Teilnahme freiwillig.

Die Entwicklung der DeGIR-Datenbank ist von unserem Kollegen Lothar Heuser nicht zu trennen. Er hat beginnend in den 1980er Jahren hier Pionierarbeit geleistet. Angefangen mit einer losen Blattsammlung entwickelte sich die Datenbank zunächst über die händische Übertragung der Interventionsdaten in eine Statistik-Software an zentralem Ort in Bochum-Langendreer, zu einer dezentralen Eingabe der Daten am Ort der Intervention, nach Versand einer entsprechenden Software per $\mathrm{CD}$, die wiederum rückgesendet und ausgewertet werden musste. Die Datenerfassung, internetbasiert über einen zentralen Server, wurde im Jahr 2005 aufgelegt. Seitdem hat sich Software als lernendes System stetig weiterentwickelt. Lothar Heuser ist auch nach seinem Ausscheiden als Institutsdirektor in Bochum-Langendreer der Sache treu geblieben. Als DeGIR-QS-Beauftragter und Vorsitzender der DeGIR-OS-Lenkungsgruppe sowie Mitglied des DeGIR-Vorstands steuert er weiterhin die Geschicke der DeGIR-Software.

Der $§ 137$ des Fünften Buchs Sozialgesetzbuch (SGB V) schreibt Maßnahmen zur Qualitätssicherung vor. Die DGNR hat 2012 unter dem damaligen DGNR-Präsidenten Olav Jansen ein entscheidendes Signal dafür gegeben, dass QS in INR erfolgreich umgesetzt wird. 


\section{Einhaltung ethischer Richtlinien}

Interessenkonflikt W. Weber und A. Berlis geben an, dass kein Interessenkonflikt besteht.

Dieser Beitrag beinhaltet keine Studien an Menschen oder Tieren.

\section{Literatur}

1. Berkhemer OA, Fransen PS, Beumer D, van den Berg LA, Lingsma HF, Yoo AJ, Schonewille WJ, Vos JA, Nederkoorn PJ, Wermer MJ, van Walderveen MA, Staals J, Hofmeijer J, van Oostayen JA, Lycklama Ã Nijeholt GJ, Boiten J, Brouwer PA, Emmer BJ, de Bruijn SF, van Dijk LC, Kappelle LJ, Lo RH, van Dijk EJ, de Vries J, de Kort PL, van Rooij WJ, van den Berg JS, van Hasselt BA, Aerden LA, Dallinga RJ, Visser MC, Bot JC, Vroomen PC, Eshghi O, Schreuder TH, Heijboer RJ, Keizer K, Tielbeek AV, den Hertog HM, Gerrits DG, van den Berg-Vos RM, Karas GB, Steyerberg EW, Flach HZ, Marquering HA, Sprengers ME, Jenniskens SF, Beenen LF, van den Berg R, Koudstaal PJ, van Zwam WH, Roos YB, van der Lugt A, van Oostenbrugge RJ, Majoie CB, Dippel DW; MR CLEAN Investigators. A randomized trial of intraarterial treatment for acute ischemic stroke. N Engl J Med. 2015;372:11-20.

2. Campbell BC, Mitchell PJ, Kleinig TJ, Dewey HM, Churilov L, Yassi N, Yan B, Dowling RJ, Parsons MW, Oxley TJ, Wu TY, Brooks M, Simpson MA, Miteff F, Levi CR, Krause M, Harrington TJ, Faulder KC, Steinfort BS, Priglinger M, Ang T, Scroop R, Barber PA, McGuinness B, Wijeratne T, Phan TG, Chong W, Chandra RV, Bladin CF, Badve M, Rice H, de Villiers L, Ma H, Desmond PM, Donnan GA, Davis SM; EXTEND-IA Investigators. Endovascular therapy for ischemic stroke with perfusionimaging selection. N Engl J Med. 2015;372:1009-18.
3. Goyal M, Demchuk AM, Menon BK, Eesa M, Rempel JL, Thornton J, Roy D, Jovin TG, Willinsky RA, Sapkota BL, Dowlatshahi D, Frei DF, Kamal NR, Montanera WJ, Poppe AY, Ryckborst KJ, Silver FL, Shuaib A, Tampieri D, Williams D, Bang OY, Baxter BW, Burns PA, Choe H, Heo JH, Holmstedt CA, Jankowitz B, Kelly M, Linares G, Mandzia JL, Shankar J, Sohn SI, Swartz RH, Barber PA, Coutts SB, Smith EE, Morrish WF, Weill A, Subramaniam S, Mitha AP, Wong JH, Lowerison MW, Sajobi TT, Hill MD; ESCAPE Trial Investigators. Randomized assessment of rapid endovascular treatment of ischemic stroke. N Engl J Med. 2015;372:1019-30.

4. Saver JL. Swift prime results. Oral presentation. Int Stroke Conference. Nashville, TN, 2015.

5. Heuser L, Arnold CN, Morhard D, Gross-Fengels W, Bücker A. [Quality report 2011 of the German Society of Interventional Radiology (DeGIR) - report about treatment quality of minimal invasive procedures]. Rofo. 2012 184(6):570-6.

6. Heuser LJ, Arnold CN, Morhard D, Köhler M, Gross-Fengels W, Bücker A. [Quality report 2011 of the German Society of Interventional Radiology (DeGIR) - part 2. Endovascular treatment of aortic aneurysms (EVAR)]. Rofo. 2013 185(8):709-19. 